\title{
Self-reported Vision Impairment and Subjective Well- being in Older Adults: A Longitudinal Mediation Analysis
}

\author{
Xiaoling Xiang, PhD, MSW, ${ }^{1}$ Vicki A. Freedman, $\mathrm{PhD},{ }^{2}$ Khushali Shah, BA, ${ }^{3,4}$ Rita X. Hu, \\ BA, ${ }^{1}$ Brian C. Stagg, MD, MS, ${ }^{3,5}$ and Joshua R. Ehrlich, MD, MPH ${ }^{3,6, *, \bullet}$
}

${ }^{1}$ School of Social Work, ${ }^{2}$ Institute for Social Research, and ${ }^{3}$ Department of Ophthalmology and Visual Sciences, Center for Eye Policy and Innovation, University of Michigan, Ann Arbor. ${ }^{4}$ Miller School of Medicine, University of Miami, Florida. ${ }^{5}$ Duke Eye Center, Durham, North Carolina. ${ }^{6}$ Institute for Healthcare Policy and Innovation, University of Michigan, Ann Arbor.

*Address correspondence to: Joshua R. Ehrlich, MD, MPH, 1000 Wall Street, Ann Arbor, MI 48105. E-mail: joshre@med.umich.edu

Received: February 18, 2019; Editorial Decision Date: June 2, 2019

Decision Editor: Jay Magaziner, MSHyg, PhD

\begin{abstract}
Background: Vision impairment (VI) in older adults is associated with declines in well-being. However, the pathways through which poor vision leads to declines in well-being have not been well-described. The purpose of this study was to determine whether activity limitations and social participation restrictions mediate the impact of self-reported VI on subjective well-being.

Methods: The National Health and Aging Trends Study (NHATS) is a nationally representative longitudinal study of Medicare beneficiaries 65 and older that includes detailed measures of the disablement process. A longitudinal mediation model was conceptualized linking selfreported VI and subjective well-being. Structural equation modeling was used to test the mediating effects of activity limitations and social participation restrictions while adjusting for relevant covariates.

Results: The final sample included 5,431 respondents. At baseline, 8.0\% of Medicare beneficiaries had self-reported VI. Subjective well-being scores were significantly lower among respondents with self-reported VI (15.7; 95\% confidence interval [CI]: 15.2, 16.2) compared with those without VI (17.6; 95\% CI: 17.5, 17.7). Self-reported VI had a significant indirect effect on subjective well-being through limiting mobility $(\beta=-0.04 ; 95 \%$ CI: $-0.07,-0.03)$ and household activities $(\beta=-0.05 ; 95 \%$ CI: $-0.08,-0.03)$, but not self-care limitations $(\beta=0.0 ; 95 \%$ $\mathrm{CI}=0.0,0.0)$ or participation restrictions $(\beta=0.0 ; 95 \% \mathrm{CI}=-0.01,0.00)$. Total indirect effects from all mediation paths accounted for $42 \%$ of the effect of VI on well-being.

Conclusions: Mobility and household activity limitations are significant mediators that explain a considerable portion of the impact of poor vision on well-being. Interventions to promote successful accommodation may result in greater overall well-being for older adults with poor vision.
\end{abstract}

Keywords: Structural equation model, NHATS, Activity limitation, Social participation, Blind

Blindness and vision impairment (VI) affect $9 \%$ of adults over age 65 in the United States, and the prevalence of these conditions is expected to more than double over the next three decades due to aging of the population and increased life expectancy $(1,2)$. Poor vision is associated with an increase in falls, depression, social isolation, cognitive decline, and decreased independence (3-6).

A growing body of literature has linked VI at older ages to declines in functioning and well-being, broadly defined (7-13). The Salisbury Eye Evaluation, a population-based longitudinal study, has provided some important insights (14-17). This study showed that refractive and nonrefractive VI resulted in limitations in mobility, near vision tasks, and driving, but that the effect was greater for nonrefractive VI (14). The researchers also used robust analytic techniques, including structural equation modeling (SEM) to test for longitudinal mediation, which showed over the 8 -year study period that visual decline led to a significantly increased hazard of mortality through increases in household activity limitations (15). In addition, the investigators used growth curve models, a method closely related to SEM, to study the longitudinal trajectories of older adults with VI and showed that poor vision is longitudinally associated with both depressive symptoms (16) and cognitive decline (17). 
Yet, the mechanisms through which VI influences overall well-being for older adults are not well understood. In a small sample of older adults with age-related macular degeneration, Rovner and Casten (11) found that the association between VI and depressive symptoms decreased with adjustment for activity limitations. In one of the only national studies that examined the factors mediating the association between VI and subjective well-being (SWB), Brown and Barret (10) analyzed data from the Americans' Changing Lives Study. They found that activity limitations, socioeconomics, social resources, and psychological resources all significantly mediated the relationship of self-reported VI with life satisfaction and depressive symptoms and that self-efficacy had the largest mediating effect (10). However, that study relied on data that are now more than 30 years old and may not represent the current generation of older U.S. adults. Additionally, measures of activity limitations and SWB in that study captured only a very limited range of disablement (18) and well-being (19) concepts.

More recent conceptualizations of the disablement process recognize that impairments may lead to activity limitations and participation restrictions, which can in turn have an adverse impact on well-being for older adults (20), and that this process may be influenced by behavioral adaptations such as the use of assistive devices (18). Conceptualization of SWB has also evolved from a relatively limited focus on symptomology (eg, depressive symptoms) to recognition of the importance of both hedonic (eg, happiness) and eudaimonic (eg, flourishing) characteristics (19). To date, analyses have not attempted to identify the pathways that connect VI to measures of SWB that capture both hedonic and eudaimonic domains.

The National Health and Aging Trends Study (NHATS) provides an opportunity to examine mediators between self-reported VI and SWB in a contemporary, nationally representative cohort of older U.S. adults. Unlike prior studies, NHATS distinguishes between how self-care and mobility activities (corresponding to activities of daily living [ADLs]) and household activities (corresponding to instrumental activities of daily living [IADLs]) are carried out, and the extent to which health and functioning limit social participation (20). The conceptualization of disability in NHATS also accounts for behavioral adaptations to changes in capacity.

The current study, using SEM, identified and quantified the direct and indirect effects of VI on SWB. In doing so, the study tests the hypothesis that self-reported VI influences SWB through its indirect effects on both activity limitations and restrictions in social participation.

\section{Method}

\section{Participants}

NHATS is a nationally representative, longitudinal study of Medicare beneficiaries aged 65 and older (www.NHATS.org). The baseline NHATS sample was initially interviewed in 2011 and the first replenished sample was added in 2015. At the time of study enrollment, all respondents were living in settings other than nursing homes.

The present study included data from three survey rounds (20152017); these data were chosen to allow for longitudinal mediation analysis using the most up-to-date data available. A total of 7,499 participants were alive, living in settings other than nursing homes at the time of study enrollment, and responded to the 2015 interview. Excluding participants with missing data on VI status in 2015 $(n=42)$ and participants who resided in nursing homes $(n=81)$, died $(n=749)$, did not respond $(n=57)$, or were lost to follow-up ( $n=1,139$ ) by 2017 , the study sample consisted of 5,431 individuals. The University of Michigan Institutional Review Board deemed this study exempt since it consisted of secondary analyses of publically available data.

\section{Measures}

\section{Vision impairment}

A participant was classified as having self-reported VI if they reported that they were blind or could not see across the street and/or read newspaper print, even with glasses. As the primary exogenous variable, VI status was obtained from the 2015 survey.

\section{Hierarchy of activity limitations}

NHATS measured activity limitations in the following self-care and mobility-related activities: eating, dressing, toileting, bathing, going outside, getting around inside, and getting out of bed. NHATS also measured limitations in selected household activities, including: doing laundry, shopping for groceries, making hot meals, paying bills and banking, and keeping track of medications.

For each activity, participants first reported use of devices or environmental modifications and assistance received performing the activity during the last month. Respondents who ever performed the activity without help then reported on difficulty doing the activity alone (with the particular devices or environmental modifications named earlier, if used). For activities other than getting out of bed, toileting, and eating, participants also reported whether they reduced the frequency of performing the activity compared with a year ago.

A previously validated four-level hierarchical classification scheme (21) was used for each of these activities: (i) fully-able: has no limitation in the ability to carry out activities (performs without devices, reduced frequency, difficulty, or assistance); (ii) successful accommodation: accommodates for limitations by using devices (for self-care and mobility activities) or by performing activities less frequently, but without difficulty or assistance; (iii) difficulty: difficulty performing activities alone even with accommodations but receives no assistance; and (iv) assistance: receives assistance from another person (due to health or functioning for household activities) or does not do the particular activity. For each set of activities (mobility, self-care, and household activities), a summary measure was created in which higher scores indicated greater limitations. Scores ranged from 4 to 16 for the self-care domain, 3 to 12 for the mobility domain, and 5 to 20 for the household domain.

\section{Social participation restrictions}

Respondents were asked to rate their preferences for the following activities from not so important, somewhat important, to very important: visiting in person with friends or family; attending religious services; participating in clubs, classes, or other organized activities; and going out for enjoyment. A score of 1 was assigned to each activity if the participant valued the activity (very or somewhat important) and their health or functioning kept them from doing the activity in the last month (18). A summary score of participation restriction across all activities (0-4) was used in the analysis, with a higher score indicating greater extent of restriction.

\section{Subjective well-being}

NHATS includes four items reflecting positive and negative emotions (frequency of feeling cheerful, bored, full of life, or upset in the last month on a five-point Likert scale) and three items reflecting 
self-realization (extent of disagreement with statements about purpose in life, self-acceptance, and environmental mastery on a 3-point Likert scale) (22). Negative items were reverse coded and ratings on the individual items were summed to create a total well-being score, ranging from 0 to 22 (Cronbach's alpha $=0.74$ ), with a higher score indicating better SWB. The scale was analyzed using confirmatory factor analysis, which demonstrated good model fit and satisfactory factor loadings for a single factor model (18).

\section{Covariates}

A parsimonious set of demographic, socioeconomic, psychosocial, and illness factors that were known to be associated with both VI and well-being $(3,4,9,23,24)$ were included as covariates. Demographic factors included age groups in 5 -year intervals, sex, and race/ethnicity. Socioeconomic factors included educational attainment, family income in quartiles, and marital status. An indicator of elevated depressive symptoms, defined as scoring $\geq 3$ on the 2-item Patient Health Questionnaire (PHQ-2) (25), was included as a psychosocial factor. Illness status was captured in the count of self-reported physician diagnoses of eight chronic conditions (hypertension, heart disease, arthritis, osteoporosis, diabetes, lung disease, stroke, and cancer). The NHATS classification of dementia status (no dementia, possible dementia, and probable dementia) was included, which was based on a self-reported diagnosis of dementia or Alzheimer's disease, an AD8 Dementia Screening Interview, and cognitive tests (26). An indicator of ever having had cataract surgery was also included. All covariates were measured as of 2015, regardless of whether respondents entered the study with the original or replenished sample.

\section{Data Analyses}

Structural equation modeling

SEM was used to test the mediation model linking self-reported VI to SWB through activity limitations and participation restrictions (Figure 1) based on the approach recommended by Hayes (27). The measurement model of SWB with seven indicators was fit using confirmatory factor analysis and then entered into a full SEM. To

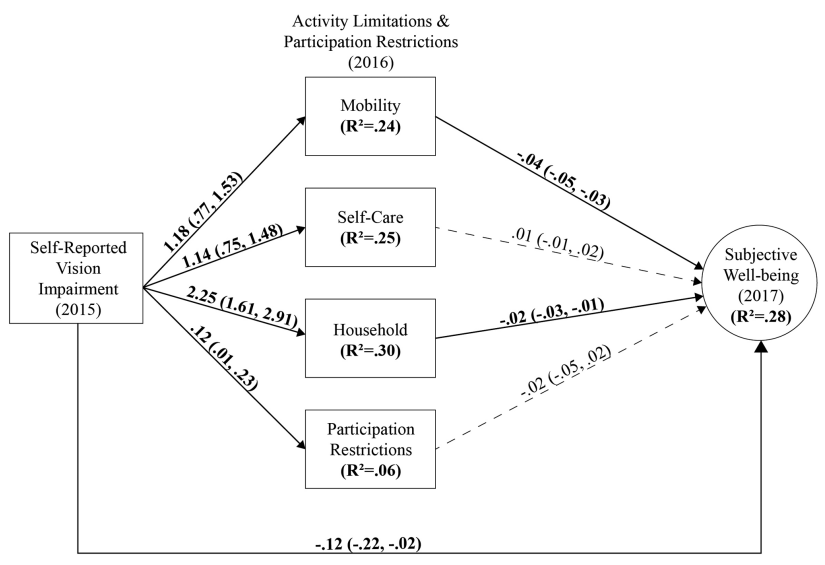

Figure 1. Structural equation model of the relationship between self-reported vision impairment and subjective well-being. Unstandardized estimates for the main paths of interest in the mediation model illustrate the relationship between self-reported vision impairment and subjective well-being. Bootstrap 95\% confidence intervals are provided. Estimates in bold are statistically significant at $p \leq .05$ and dashed lines represent nonsignificant effects. establish a temporal order, the assessment of VI took place in 2015, the assessment of the mediators (mobility, self-care, and household activity limitations, and participation restrictions) in 2016, and SWB in 2017.

The main paths of interest included the direct effects of VI on SWB and indirect effects through activity limitations and participation restrictions. In addition, SWB was regressed on the aforementioned covariates. The four mediators (three activity limitation measures and participation restrictions) were also regressed on covariates but only retained significant paths in the final model to maintain a parsimonious model. A good-fitting SEM has a a root mean square error of approximation (RMSEA) <.06, comparative fit index $(\mathrm{CFI})>.95$, and standardized root mean square residual (SRMR) <.08 (28). After establishing an unweighted model with good model fit indices, the model was re-estimated using the 2017 NHATS sample design variables (ie, strata, cluster, and analytical weights) and a Bootstrap resampling method for variance estimation (29) to generate weighted estimates. Analyses were conducted in Mplus 8 (Muthén \& Muthén, Los Angeles, CA).

A minimal amount of missing data for SWB $(4.9 \%$ of the sample) occurred due to partial response and the use of proxies (only selfrespondents answered questions about SWB). Missing data were treated using a full information maximum likelihood (FIML) method, wherein all available information was used to estimate the model.

\section{Sensitivity analyses}

Sensitivity analyses were performed to check the robustness of study findings against different methods for handling missing data. The SEM shown in Figure 1 was re-estimated $(n=5,192)$ excluding proxies. The model was also estimated with all persons who reported VI status in 2015, including those who resided in nursing homes, died, or were lost to follow-up $(n=7,527)$ with missing data imputed by carrying forward the last observation.

\section{Results}

In 2015, $8.0 \%$ of community-dwelling Medicare beneficiaries were classified as having VI, which corresponded to more than 3.5 million persons in the U.S. population. Table 1 presents weighted characteristics of the study population at baseline, stratified by VI status. Compared with those without self-reported VI, respondents with VI were more likely to be older, female, nonwhite, less educated, not married or cohabiting, have lower family income, elevated depressive symptoms, dementia, more medical commodities, and have received cataract surgery $(p<.001$ for all comparisons). Respondents with self-reported VI scored an average of 6.5 on the mobility limitations scale $(95 \%$ confidence interval [CI]: 6.1, 6.9), 7.4 on the self-care limitations scale (95\% CI: 7.0, 7.9), 10.4 on the household activity limitations scale $(95 \%$ CI: 9.6, 11.1), and had an average of 0.5 participation restrictions, all of which were significantly higher compared to respondents without VI. Respondents with self-reported VI had significantly lower SWB $(15.7 ; 95 \%$ CI: $15.2,16.2)$ than those without VI (17.6; 95\% CI: 17.5, 17.7).

Confirmatory factor analysis revealed good fit for the measurement model based on RMSEA $=0.032$, CFI $=0.952$, and SRMR $=0.02$. The unweighted structural model also had a good model fit based on RMSEA $=0.032, \mathrm{CFI}=0.951$, and $\mathrm{SRMR}=0.02$. The weighted structural model had SRMR $=0.02$, whereas other model fit indices were unavailable due to the application of Bootstrap resampling methods and sample weighting. 
Table 1. Baseline Sample Characteristics by Vision Impairment Status $(N=5,431)^{*}$

\begin{tabular}{|c|c|c|}
\hline Characteristic & No Self-reported Vision Impairment & Self-reported Vision Impairment \\
\hline \multicolumn{3}{|l|}{ Age groups (\%) } \\
\hline $65-69$ years & $31.9(30.2,33.7)$ & $25.2(18.8,33.0)$ \\
\hline $70-74$ years & $28.1(26.5,29.7)$ & $22.5(17.9,27.9)$ \\
\hline $75-79$ years & $18.8(17.9,19.7)$ & $21.2(16.5,26.8)$ \\
\hline $80-84$ years & $12.1(11.3,13.0)$ & $11.4(8.8,14.6)$ \\
\hline $85-89$ years & $6.2(5.7,6.9)$ & $12.0(9.4,15.2)$ \\
\hline 90 years or over & $2.9(2.5,3.3)$ & $7.6(6.0,9.6)$ \\
\hline \multicolumn{3}{|l|}{$\operatorname{Sex}(\%)$} \\
\hline Female & $54.5(52.9,56.1)$ & $64.8(59.6,69.7)$ \\
\hline Male & $45.5(43.9,47.1)$ & $35.2(30.3,40.4)$ \\
\hline \multicolumn{3}{|l|}{ Race/ethnicity (\%) } \\
\hline White, non-Hispanic & $79.5(77.1,81.7)$ & $64.1(57.0,70.6)$ \\
\hline Black, non-Hispanic & $7.7(6.8,8.8)$ & $10.5(8.2,13.2)$ \\
\hline Hispanic & $6.4(5.1,8.1)$ & $16.9(12.7,22.1)$ \\
\hline Other & $6.4(5.4,7.5)$ & $8.6(4.6,15.5)$ \\
\hline \multicolumn{3}{|l|}{ Education $(\%)$} \\
\hline Less than high school & $14.5(13.1,16.1)$ & $32.2(27.4,37.4)$ \\
\hline High school & $24.5(22.4,26.7)$ & $31.6(26.6,36.9)$ \\
\hline Some college, no degree & $23.6(21.9,25.4)$ & $18.1(14.5,22.4)$ \\
\hline College graduate & $37.4(34.5,40.3)$ & $18.1(14.0,23.1)$ \\
\hline Family income in 2015 (\$) (mean) & $73,273(61,179,85,366)$ & $40,340(33,028,47,653)$ \\
\hline Married or living with a partner $(\%)$ & $59.2(57.5,61.0)$ & $44.0(38.5,49.6)$ \\
\hline Depression symptoms $(\%)$ & $9.9(9.0,11.0)$ & $25.7(20.4,31.7)$ \\
\hline Chronic disease count (mean) & $2.2(2.2,2.3)$ & $2.8(2.6,3.0)$ \\
\hline \multicolumn{3}{|l|}{ Dementia status (\%) } \\
\hline No dementia & $89.4(88.5,90.3)$ & $69.3(64.1,73.9)$ \\
\hline Possible dementia & $6.3(5.7,7.1)$ & $11.9(9.0,15.7)$ \\
\hline Probable dementia & $4.2(3.7,4.9)$ & $18.8(14.8,23.6)$ \\
\hline Ever had cataract surgery (\%) & $38.3(36.5,40.0)$ & $53.4(48.0,58.7)$ \\
\hline Mobility limitations (mean) & $4.1(4.1,4.2)$ & $6.5(6.1,6.9)$ \\
\hline Self-care limitations (mean) & $5.5(5.4,5.6)$ & $7.4(7.0,7.9)$ \\
\hline Household limitations (mean) & $6.6(6.5,6.7)$ & $10.4(9.6,11.1)$ \\
\hline Participation restrictions (mean) & $0.2(0.2,0.3)$ & $0.5(0.4,0.6)$ \\
\hline Subjective well-being (mean) & $17.6(17.5,17.7)$ & $15.7(15.2,16.2)$ \\
\hline
\end{tabular}

Note: All bivariate comparisons were significant at $p<.001$.

*NHATS 2017 complex survey design features used for generating weighted estimates using Taylor linearization for variance estimation.

Baseline self-reported VI had a statistically significant direct and negative association with SWB 2 years later $(\beta=-0.12 ; 95 \% \mathrm{CI}$ : $-0.21,-0.04)$ after adjusting for covariates. Baseline self-reported VI was significantly associated with activity limitations 1 year later after adjusting for covariates. Specifically, self-reported VI was associated with a 1.18 points increase $(95 \% \mathrm{CI}: 0.77,1.53)$ in mobility limitations, 1.14 points increase $(95 \% \mathrm{CI}: 0.75,1.48)$ in self-care limitations, 2.25 points increase $(95 \%$ CI: $1.61,2.91)$ in household activity limitations, and 0.12 points increase $(95 \%$ CI: $0.01,0.23)$ in participation restrictions. Higher levels of mobility $(\beta=-0.04 ; 95 \%$ CI: $-0.05,-0.03)$ and household activity limitations $(\beta=-0.02 ; 95 \%$ CI: $-0.03,-0.01)$ in 2016 were significantly associated with lower levels of SWB 1 year later, whereas the paths from self-care limitations and participation restrictions to SWB were not statistically significant after adjusting for covariates. The SEM model explained $28 \%$ of the variance in SWB, $24 \%$ in mobility, $25 \%$ in self-care, and $30 \%$ in household activity limitations, as well as $6 \%$ in social participation restrictions.

Results from the mediation analysis supported activity limitations as mediators of the relationship between VI and subjective well-being (Table 2). Self-reported VI had a significant indirect effect on SWB through mobility limitations $(\beta=-0.04 ; 95 \% \mathrm{CI}$ : $-0.07,-0.03)$ and household activity limitations $(\beta=-0.05 ; 95 \%$
CI: $-0.08,-0.03)$, whereas the indirect effects of VI through selfcare limitations $(\beta=0.01 ; 95 \%$ CI: $-0.00,0.01)$ and participation restrictions $(\beta=-0.00 ; 95 \% \mathrm{CI}:-0.01,0.01)$ were not statistically significant. The total indirect effects from all mediation paths linking self-reported VI to SWB were $\beta=-0.086$ (95\% CI: $-0.126,-0.054)$, accounting for $42 \%$ of the total effect of VI on SWB.

\section{Sensitivity Analyses}

Results from the SEM excluding proxies produced nearly identical results to the main analysis that included proxies. Analysis from the model including all respondents who reported VI status in 2015 also produced comparable results; for example, the total indirect effect of self-reported VI on SWB was $\beta=-0.101(p<.05)$ or $45 \%$ of the total effect.

\section{Discussion}

Using longitudinal data from a nationally representative cohort of older U.S. adults, this study found that self-reported VI was significantly associated with lower levels of SWB. Moreover, nearly half of the effect of VI on SWB was accounted for by the mediating pathway that included household activity and mobility limitations. 
Table 2. Total, Direct, Total Indirect, and Specific Indirect Effects of Vision Impairment on Subjective Well-being

\begin{tabular}{llll}
\hline Self-Reported Vision Impairment $\rightarrow$ Subjective Well-being & $\beta^{*}$ & $95 \%$ CI & Beta \\
\hline Total effects & -0.207 & $-0.303,-0.114$ & -0.112 \\
Direct effect & -0.121 & $-0.205,-0.039$ & -0.065 \\
Total indirect effects & -0.086 & $-0.126,-0.054$ & -0.047 \\
VI $\rightarrow$ Mobility limitations $\rightarrow$ SWB & -0.043 & $-0.068,-.0 .025$ & -0.023 \\
VI $\rightarrow$ Self-care limitations $\rightarrow$ SWB & 0.008 & $-0.008,0.029$ & -0.004 \\
VI $\rightarrow$ Household activity limitations $\rightarrow$ SWB & -0.049 & $-0.083,-0.026$ & -0.026 \\
VI $\rightarrow$ Participation restrictions $\rightarrow$ SWB & -0.002 & $-0.010,0.001$ & -0.001 \\
\hline
\end{tabular}

Note: $\mathrm{CI}$ = confidence interval; SWB = subjective well-being; $\mathrm{VI}$ = self-reported vision impairment.

" $\beta$ represents unstandardized coefficients with $95 \%$ bootstrap confidence intervals presented.

This study makes several important contributions. First, it employed new data from NHATS that offer a nuanced set of constructs for studying the impact of poor vision on late-life disablement and well-being. Second, this study is among the first to show that household activity and mobility limitations are significant factors linking self-reported VI to decreased well-being. Finally, it suggests that interventions to help older adults with VI successfully accommodate for activity limitations may have a positive effect on overall well-being.

In this study, baseline self-reported VI was significantly associated with lower SWB 2 years later, and $42 \%$ of this effect was accounted for by respondents' intervening activity limitation and social participation status. The significant mediators in this model were household activity and mobility limitations, which together accounted for the indirect effects in the SEM. Each of these pathways represents a potential therapeutic target for future vision rehabilitation programs designed to optimize the well-being of older adults with VI.

The total effect of self-reported VI on SWB in this study was -0.207 (95\% CI: $-0.303,-0.114)$. It is not possible to make a direct comparison with results from other studies that have used the NHATS SWB scale (30), since using a distinct set of covariates, sample restriction criteria, and analytic strategies may have a large effect on regression coefficients. However, in this study, the magnitude of the effect of VI on SWB was larger than the effect of probable dementia ( $\beta=-0.133 ; 95 \%$ CI: $-0.108,-0.084)$ but smaller than the effect of clinically significant depressive symptoms $(\beta=-0.537 ; 95 \%$ CI: $-0.499,-0.484$ ) on SWB.

This study adds to the small body of research that has sought to describe the pathways linking VI with well-being declines in older adults $(10,11)$. Consistent with prior studies, this study found that measures of activity limitations are significant factors in the relationship between self-reported VI and well-being. Unlike prior studies, however, this study quantified the importance of specific pathways using SEM. In doing so, two central mediators were identified-mobility and household activity limitations-that accounted for $21 \%$ and $24 \%$ or the total effect of VI on SWB, respectively. Although VI was also associated with increased self-care limitations and participation restrictions, these did not significantly impact respondents' SWB. One possible explanation for this finding is that self-care limitations tend to occur late in the disablement process and affect the oldest old, a group also tends to report better hedonic measures of well-being (31) (pp. 251-253, 278-282) (32). Participation restrictions only had a small, albeit statistically significant, association with self-reported VI and may have been largely driven by covariates in the model.

The current study recognizes that not all activity limitations are equivalent in terms of their impact on well-being. The validated activity limitation hierarchies (21) utilized in this study distinguish older adults who successfully accommodate through the use of assistive devices and environmental modifications from those who report difficulty or receive assistance from others. This study also considered activities beyond those essential for independent living. Many prior studies have taken a less nuanced approach to understanding the adverse consequences of VI. For example, among the numerous studies that have reported increased activity limitations in older adults with VI $(11,33,34)$, few have accounted for whether respondents successfully accommodate for declines in functioning, perform activities less frequently, or perform an activity only with considerable difficulty. In the current study, the activity limitation hierarchies were a significant mediator of the relationship between VI and SWB, which suggests that interventions to promote successful accommodation could move individuals higher on these hierarchies, which may improve SWB.

Several prior studies have shown that adults with VI are more likely to have restricted social participation compared with their peers (12,34-36). For example, in a longitudinal study of adults in England, Matthews and colleagues (12) reported that worsening self-reported vision predicted decreases in social engagement and well-being, though they did not test the mediating pathways between these variables. The few studies that have explored the mediating effect of social activity on the association between VI and well-being have focused on social support rather than social participation, and results have been mixed $(19,37)$. The current study contributes to this debate by demonstrating that self-reported VI was significantly and independently associated with participation restrictions, but that this did not mediate the association between VI and SWB.

This study had several limitations. Data were self-reported and therefore may be subject to recall and social desirability biases. There may have also been selection bias, wherein sicker respondents were more likely to be lost to follow-up or die, which could have biased results toward the null hypothesis since they may have been at greater risk for poor vision, activity limitations, and SWB decline (3). Though self-reported vision status has been widely used in prior population-based studies $(37,38)$, there could be a confounding effect of personality type. Future work should corroborate this study's findings using objective measures of visual function like visual acuity and contrast sensitivity. This study did not examine how changes in mediators over time relate to changes in SWB, though this may be an important next step. Finally, questions used to measure self-reported VI in NHATS were related to central vision; this could have led to misclassification of those with isolated peripheral vision loss, which would likely have biased results toward the null hypothesis.

A strength of this study was the use of a large nationally representative sample with validated measures of key constructs such as 
the activity limitation and participation restriction hierarchies and SWB. Another major strength was the use of SEM to test for multiple mediation. SEM allows for both latent and observed variables and is designed in part to test complicated mediation models in a single analysis involving multiple independent variables, mediators, and outcomes (39). In contrast, standard regression relies on ad hoc methods that combine results from two or more equations to derive the asymptotic variance for inference about indirect and total effects, which can be problematic, particularly in the presence of missing data (40). Another advantage of SEM over standard regression methods is that SEM analyses provide model fit information, which can be seen as evidence for the plausibility of assumptions about causality (40).

This study demonstrates that mobility and household activity limitations are significant mediators that explain the adverse impact of self-reported VI on well-being over time. Even after adjusting for important covariates like depressive symptoms and dementia status, nearly half of the effect of poor vision on well-being was explained by these mediators. This finding suggests the importance of developing comprehensive vision rehabilitation strategies to interrupt these pathways. Interventions like assistive devices and environmental modifications that transition older adults to higher positions on the activity limitations hierarchies may have a considerable positive impact on SWB. The burden of VI among older adults is large and is projected to grow over the coming decades. Accordingly, addressing the consequences of vision loss is vital for promoting the well-being of older individuals and an aging population.

\section{Funding}

This work was supported by grants from the National Eye Institute at the National Institutes of Health (K23 EY027848 to J.R.E.); the Michigan Center on the Demography of Aging (MiCDA) at the University of Michigan to J.R.E. through a core grant from the National Institute on Aging at the National Institutes of Health (P30 AG012846); the University of Michigan Older Americans Independence Center to X.X. through a core grant from the National Institute on Aging at the National Institutes of Health (P30 AG024824); the National Institute on Aging (U01AG032947); the Lighthouse Guild to the Department of Ophthalmology and Visual Sciences at the University of Michigan; an unrestricted grant from Research to Prevent Blindness to the Department of Ophthalmology and Visual Sciences at the University of Michigan. The funding organizations had no role in the design and conduct of the study; collection, management, analysis, and interpretation of the data; and preparation, review, or approval of the article.

\section{Author Contributions}

X.X. was involved in conception/design, analysis, and drafting the article. V.A.F. and R.H. was involved in interpretation of data and critical revision of the article. K.S. was involved in acquisition and analysis of data and critical revision of the article. B.C.S. was involved in conception/design and critical revision of the article. J.R.E. was involved in conception/design, interpretation of data and drafting the article.

\section{Conflict of Interest}

V.A.F. is Co-Principal Investigator of the National Health and Aging Trends Study. No author has any other relevant conflicts of interest to declare.

\section{References}

1. Wittenborn JS, Zhang X, Feagan CW, et al.; Vision Cost-Effectiveness Study Group. The economic burden of vision loss and eye disorders among the United States population younger than 40 years. Ophthalmology. 2013;120:1728-1735. doi:10.1016/j.ophtha.2013.01.068

2. Chan T, Friedman DS, Bradley C, Massof R. Estimates of incidence and prevalence of visual impairment, low vision, and blindness in the United States. JAMA Ophthalmol. 2017;136(1):12-19. doi:10.1001/ jamaophthalmol.2017.4655

3. National Academies of Sciences, Engineering, and Medicine. Making Eye Health a Population Health Imperative. Washington, DC: The National Academies Press; 2016.

4. Ehrlich JR, Hassan SE, Stagg BC. Prevalence of falls and fall-related outcomes in older adults with self-reported vision impairment. J Am Geriatr Soc. 2019;67:239-245. doi:10.1111/jgs.15628

5. Chen SP, Bhattacharya J, Pershing S. Association of vision loss with cognition in older adults. JAMA Ophthalmol. 2017;135:963-970. doi:10.1001/ jamaophthalmol.2017.2838

6. Fenwick EK, Ong PG, Man REK, et al. Association of vision impairment and major eye diseases with mobility and independence in a Chinese population. JAMA Ophthalmol. 2016;134(10):1087-1093. doi:10.1001/ jamaophthalmol.2016.2394

7. Broman AT, Munoz B, Rodriguez J, et al. The impact of visual impairment and eye disease on vision-related quality of life in a MexicanAmerican population: proyecto VER. Invest Ophthalmol Vis Sci. 2002;43:3393-3398.

8. DeCarlo DK, Scilley K, Wells J, Owsley C. Driving habits and healthrelated quality of life in patients with age-related maculopathy. Optom Vis Sci. 2003;80:207-213. doi:10.1097/00006324-200303000-00010

9. Freeman EE, Muñoz B, West SK, Jampel HD, Friedman DS. Glaucoma and quality of life: the Salisbury Eye Evaluation. Ophthalmology. 2008;115:233-238. doi:10.1016/j.ophtha.2007.04.050

10. Brown RL, Barrett AE. Visual impairment and quality of life among older adults: an examination of explanations for the relationship. J Gerontol B Psychol Sci Soc Sci. 2011;66:364-373. doi:10.1093/geronb/gbr015

11. Rovner BW, Casten RJ. Activity loss and depression in age-related macular degeneration. Am J Geriatr Psychiatry. 2002;10:305-310. doi:10.1097/00019442-200205000-00010

12. Matthews K, Nazroo J, Whillans J. The consequences of self-reported vision change in later-life: evidence from the English Longitudinal Study of Ageing. Public Health. 2017;142:7-14. doi:10.1016/j. puhe.2016.09.034

13. Rafaely L, Carmel S, Bachner YG. Subjective well-being of visually impaired older adults living in the community. Aging Ment Health.. 2017;22(9):1223-1231. doi:10.1080/13607863.2017.1341469

14. Zebardast N, Swenor BK, van Landingham SW, et al. Comparing the impact of refractive and nonrefractive vision loss on functioning and disability: the Salisbury Eye Evaluation. Ophthalmology. 2015;122:11021110. doi:10.1016/j.ophtha.2015.02.024

15. Christ SL, Zheng DD, Swenor BK, et al. Longitudinal relationships among visual acuity, daily functional status, and mortality: the Salisbury Eye Evaluation Study. JAMA Ophthalmol. 2014;132:1400-1406. doi:10.1001/jamaophthalmol.2014.2847

16. Zheng DD, Bokman CL, Lam BL, et al. Longitudinal relationships between visual acuity and severe depressive symptoms in older adults: the Salisbury Eye Evaluation study. Aging Ment Health. 2016;20:295-302. doi:10.1080/13607863.2015.1008985

17. Zheng DD, Swenor BK, Christ SL, West SK, Lam BL, Lee DJ. Longitudinal associations between visual impairment and cognitive functioning: the Salisbury Eye Evaluation study. JAMA Ophthalmol. 2018;136:989-995. doi:10.1001/jamaophthalmol.2018.2493

18. Freedman VA, Kasper JD, Spillman BC, et al. Behavioral adaptation and late-life disability: a new spectrum for assessing public health impacts. Am J Public Health. 2014;104:e88-e94. doi:10.2105/AJPH.2013.301687

19. Centers for Disease Control and Prevention. Well-being concepts. https:// www.cdc.gov/hrqol/wellbeing.htm. Updated November 5, 2018. Accessed January 30, 2019.

20. Freedman VA. Adopting the ICF language for studying late-life disability: a field of dreams? J Gerontol A Biol Sci Med Sci. 2009;64:1172-1174; discussion 1175. doi:10.1093/gerona/glp095 
21. Gill TM, Williams CS. Evaluating distinctions in the assessment of late-life disability. J Gerontol A Biol Sci Med Sci. 2017;72:1538-1546. doi:10.1093/gerona/glx022

22. Ryff CD. Happiness is everything, or is it? Explorations on the meaning of psychological well-being. J Pers Soc Psychol.. 1989;57(6):1069-1081. doi:10.1037/0022-3514.57.6.1069

23. Stagg BC, Choi H, Woodward MA, Ehrlich JR. Association of social support network size with receipt of cataract surgery in older adults. JAMA Ophthalmol. 2018;136:423-427. doi:10.1001/ jamaophthalmol.2018.0244

24. Janz NK, Wren PA, Lichter PR, et al.; CIGTS Study Group. The Collaborative Initial Glaucoma Treatment Study: interim quality of life findings after initial medical or surgical treatment of glaucoma. Ophthalmology. 2001;108:1954-1965. doi:10.1016/S0161-6420(01)00874-0

25. Löwe B, Kroenke K, Gräfe K. Detecting and monitoring depression with a two-item questionnaire (PHQ-2). J Psychosom Res. 2005;58:163-171. doi:10.1016/j.jpsychores.2004.09.006

26. Kasper J, Freedman V, Spillman B. Classification of persons by dementia status in the National Health and Aging Trends Study. Technical Paper \#5. https://www.nhats.org/scripts/documents/NHATS_Dementia_Technical_ Paper_5_Jul2013.pdf. Updated 2013. Accessed January 30, 2019.

27. Hayes AF. Beyond Baron and Kenny: statistical mediation analysis in the new millennium. Commun Monogr. 2009;76(4):408-420. doi:10.1080/03637750903310360

28. Hooper D, Coughlan J, Mullen M. Structural equation modelling: guidelines for determining model fit. Articles 2008:2:2

29. Stapleton LM. Variance estimation using replication methods in structural equation modeling with complex sample data. Struct Equ Model. 2008;15(2):183-210. doi:10.1080/10705510801922316

30. Zahuranec DB, Skolarus LE, Feng C, Freedman VA, Burke JF. Activity limitations and subjective well-being after stroke. Neurology. 2017;89:944950. doi:10.1212/WNL.0000000000004286
31. Albert SM, Freedman VA. Public Health and Aging. 2nd ed. New York, NY: Springer Publishing Company; 2010.

32. Horne AJ, Chiew KS, Zhuang J, et al. Relating sensory, cognitive, and neural factors to older persons' perceptions about happiness: an exploratory study. J Aging Res. 2018;2018:4930385. doi:10.1155/2018/4930385

33. West CG, Gildengorin G, Haegerstrom-Portnoy G, Schneck ME, Lott L, Brabyn JA. Is vision function related to physical functional ability in older adults? J Am Geriatr Soc. 2002;50:136-145. doi:10.1046/j.1532-5415.2002.50019.x

34. Naël V, Pérès K, Carrière I, et al. Visual impairment, undercorrected refractive errors, and activity limitations in older adults: findings from the Three-City Alienor Study. Invest Ophthalmol Vis Sci.. 2017;58(4):23592365. doi:10.1167/iovs.17-21525

35. Crews JE, Campbell VA. Vision impairment and hearing loss among community-dwelling older Americans: implications for health and functioning. Am J Public Health. 2004;94:823-829. doi:10.2105/ ajph.94.5.823

36. Lamoureux EL, Hassell JB, Keeffe JE. The determinants of participation in activities of daily living in people with impaired vision. Am J Ophthalmol. 2004;137:265-270. doi:10.1016/j.ajo.2003.08.003

37. Lam BL, Lee DJ, Zheng DD, Davila EP, Christ SL, Arheart KL. Disparity in prevalence of self-reported visual impairment in older adults among U.S. race-ethnic subgroups. Ophthalmic Epidemiol. 2009;16:144-150. doi:10.1080/09286580902863007

38. Whillans J, Nazroo J. Assessment of visual impairment: the relationship between self-reported vision and 'gold-standard' measured visual acuity. Br J Vis Impair. 2014;32(3):236-248. doi:10.1177/0264619614543532

39. MacKinnon D. Introduction to Statistical Mediation Analysis. Abingdonon-Thames, UK: Routledge; 2012.

40. Gunzler D, Chen T, Wu P, Zhang H. Introduction to mediation analysis with structural equation modeling. Shanghai Arch Psychiatry. 2013;25:390-394. doi:10.3969/j.issn.1002-0829.2013.06.009 